\title{
Research on Optimization Design of Lifting Mechanism of Hot Air Drying Room Gate
}

\author{
Guang Fei Wang, Ping Yan \\ College of Mechanical Engineering, Shanghai University of Engineering Science, Shanghai, China
}

Email address:

1371897901@qq.com (Guang Fei Wang), 978538640@qq.com (Ping Yan)

To cite this article:

Guang Fei Wang, Ping Yan. Research on Optimization Design of Lifting Mechanism of Hot Air Drying Room Gate. Engineering and Applied Sciences. Vol. 2, No. 4, 2017, pp. 72-76. doi: 10.11648/j.eas.20170204.13

Received: March 22, 2017; Accepted: April 5, 2017; Published: September 5, 2017

\begin{abstract}
Artificial hand lifting device for the traditional hot air drying oven door is optimized by design UG software to provide power to the lifting device of hydraulic pump. Thus driving the working mechanism to realize linear reciprocating rotary motion. This improvement improves safety during drying efficiency and the drying room door landing, convenient on-site workers the operation, improve the operation environment.
\end{abstract}

Keywords: Drying House, Hydraulic Lifting Device, Motion Simulation

\section{Introduction}

Wood is one of the world's most widely used engineering materials. Responsible for forest protection of the ecological environment and provide timber resources two functions, facing the world's forest resources increasingly reduce the environmental and ecological problems, how to effectively use of timber resources, reduce energy consumption, improve the quality of wood products has attracted wide attention of the government. My demand China has more than the supply of wood, is a lack of forest resources of the country, how to better improve the utilization rate of wood, improve its performance, it has become an important research topic in the presence of wood science workers.

Wood drying is in the control of medium temperature and relative humidity conditions of wood heating, the water inside the wood moves to the surface, the surface water evaporation to the outside world, gradually out of the water from the wood. The wood drying is an essential link in the process of wood, is to improve the utilization ratio of timber, reduce timber loss, ensure wood the quality of the products, the important technical measures to improve the physical and mechanical properties of wood.

Wood drying is the protection and improvement of wood quality, reduce wood loss, improve the utilization ratio of timber link. The largest energy consumption at the same time also wood drying wood products in the production process, so in the wood drying, improve drying rate and quality, to reduce the energy consumption and the cost of drying is necessary [1] hot air drying is the real wood processing enterprises the use of drying equipment in the most, $80 \%$ Chinese wood using conventional drying room drying [2], which can effectively improve the work efficiency of the wood drying house is environmentally friendly and can increase the added value of product. The drying room door opening and automatic closing is a heavy influence of wood drying efficiency To be factors in the actual production, because of the workers' manual opening and closing door of the drying room drying room door closed time-consuming and not tight, the influence on the efficiency of forklift transporting timber stacking is also accompanied by the loss of a large number of drying room energy, greatly reduces the efficiency of wood drying.

In this paper, the manual manual lifting device of the traditional hot air drying chamber is optimized. Due to the rotation of the rocker is through the worm, the worm helical motion into linear motion, but in the actual operation process. The rod is time-consuming and laborious, affect the working efficiency of the drying room, after optimization in hydraulic system the main [3], the motive of the mechanical energy is converted to liquid pressure can use hydraulic pump to transfer energy through the change of liquid pressure, thus driving the working mechanism to realize linear reciprocating rotary motion, so to improve safety during drying efficiency and the drying room door landing, convenient now Field workers operation, improve the operation environment. 


\section{Hot Air Drying Oven Door Lifting Device}

The conventional drying room door in the form of a total of five different types, which are the single door or double doors hinged doors, multi folding door, multi fan hanging door, single door hanging door and single door lifting door. But in the market at present is the most common type of hanging door, this design is innovative and according to the improved hanging door single fan. As shown in Figure 1 (a), (b) shows, the traditional hot air drying oven door is opened or closed by using artificial hand-operated lifting frame, worm gear engaging device using helical motion into linear motion of the worm gear worm, in the

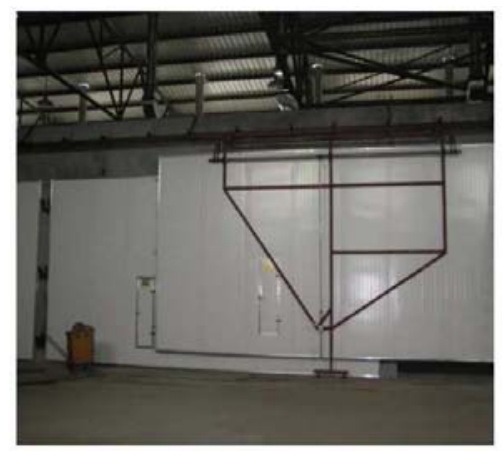

(a) baking room the door opened and closed by the lever principle. As a result of this end The full implementation of the manual, construction personnel will not only increase the labor intensity and danger and will appear in the door open when the efficiency is low, resulting in a huge loss of heat of drying room, so need to preheat the filling wood, increase unnecessary energy consumption. Also, with the drying room size is high, the the quality of the door is bigger also, manual operation is also increasing, the construction personnel in the closed door room often occurs is not easy to reset, which leads to the door are not closed tight also leads to energy loss. Based on the above points, the traditional lifting device has been unable to meet the design requirements, hydraulic hanger design Came into being.

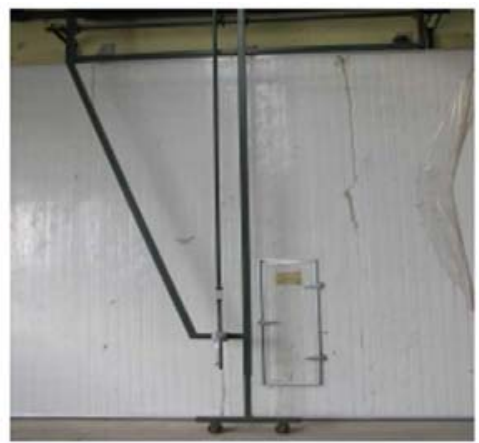

(b)

Figure 1. Hand-operated gate lifting device of ordinary drying room.

\section{Design of Hydraulic Lifting Device for New Type of Hot Air Drying Drying Door}

\subsection{Model}

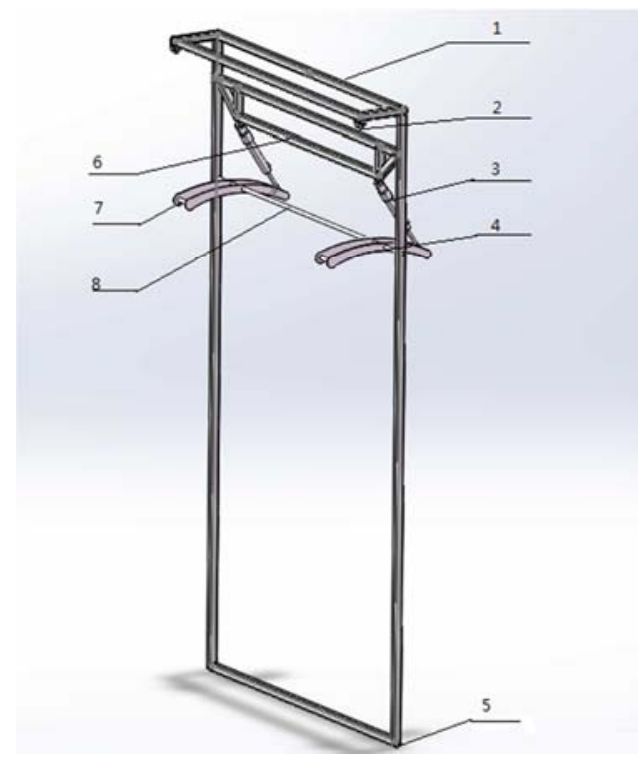

Figure 2. Hot air drying oven door structure of hydraulic lifting device.

1-Door frame; 2-Top pulley; 3-Hydraulic cylinder; 4-Crank rocker; 5-Bottom pulley; 6-Strengthening support; 7-Sleeve rod; 8-The stress of connecting rod

\subsection{Analysis of a New Type of Hydraulic Lifting Device of Movement}

The technical scheme, through the telescopic movement of the hydraulic cylinder, the force of the rod on the arc movement. Because the stress of the connecting rod and the crank rocker crank rocker and rigid fixation, and the door frame are connected by a rotary pair, the crank rocker will rotate at a certain angle. Drying house door with the crank rocker link, make changes in the displacement of crank rocker drive. Further, when the hydraulic cylinder was introduced, the crank rocker upward arc movement, drying house door was brought out, then by doing horizontal movement, opened the door, wood free into the drying room. The skeleton structure of the pulley on the part of the group the door is moved to the drying room entrance is In front of the hydraulic cylinder return crank rocker downward arc movement, drying house door was inward down, the hole was sealed plugging.

The specific implementation scheme: the hydraulic cylinder 3 through a rotating shaft and bracket reinforcement structure 6 connected through the sleeve is connected with the connecting rod. The stress force of connecting rod and crank rocker 4 rigid, crank rocker and 4 door frame structure 1 is connected by a rotary pair, a link rod drying house door lug and the crank rocker 4 . When the hydraulic cylinder 3 is launched, the force rod downward arc movement, and crank rocker rotating crank rocker 4,4 connected with the upward movement of the arc, drying house door was brought out, open the gate. The hydraulic cylinder 3 Return crank rocker 4 end downward arc movement, drying drying room door was 
inward down, the hole was sealed Blocking.

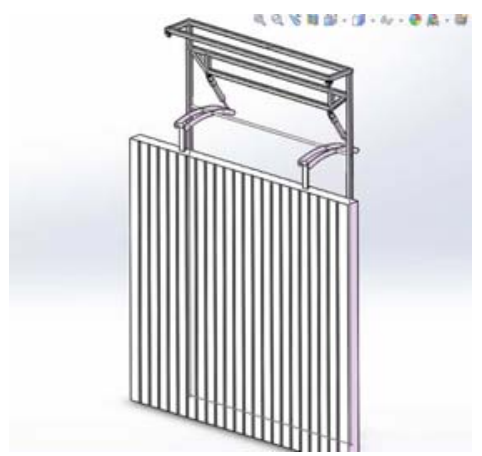

Figure 3. Drying house door hydraulic lifting device structure layout.

\subsection{The Motion Simulation Model of Hydraulic Lifting Device}

Design of lifting device, first need to determine the link in the process of lifting the door to the size of the force, namely the hydraulic cylinder output force. The drying room in the design of the gate of the weight of 0.5 tons, to ensure the mechanical requirements and safety coefficient, so take the oven weight of 1 tons, i.e.:

$$
\mathrm{F}=1 \times 10000=10000 \mathrm{~N}
$$

In order to determine the movement of the drying room door in the lifting process, this paper uses the motion simulation of NX8.5 software function, by means of simulation, the force and movement of the door to the function curve expressed, so as to get the maximum stress value in the process of movement and gate displacement amplitude. In the simulation module in dynamic analysis, in the whole process of simulation, the door frame does not produce any displacement, so the door bracket and the upper and lower pulley is provided with a fixed rod, then the link between pairs defined in the device, most sports vice rotation pair, to the center of rotation axis corresponds to the rotation of the connecting link The center vector of the direction of rotation to determine by the right-hand rule. Figure 4 is a process simulation in the screenshot, figure 5 door to the initial position, the figure 6 is entirely up to the position of gate.

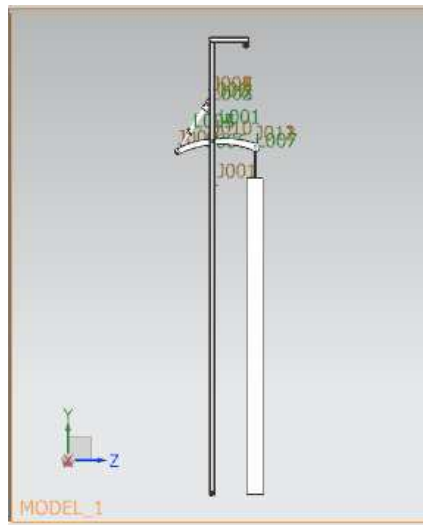

Figure 4. Drying room door lifting device simulation.

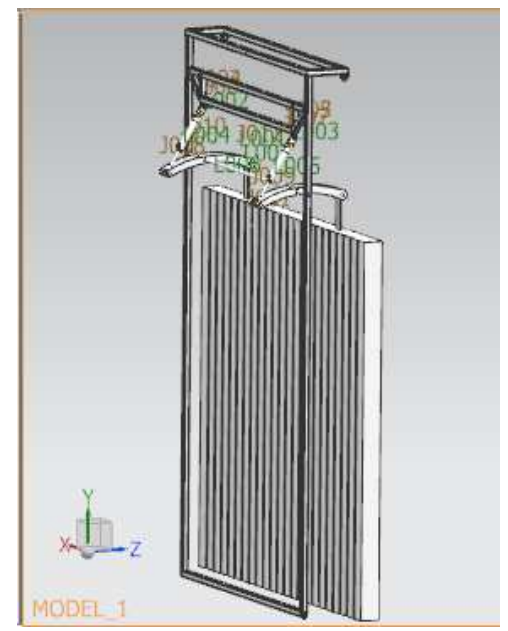

Figure 5. Drying room door to the initial position.

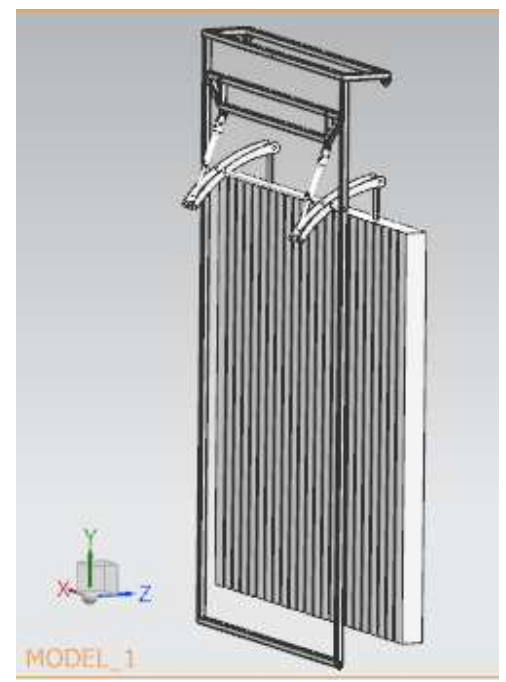

Figure 6. The end position of drying room door.

In order to obtain the force curve, the need to select the hydraulic cylinder to push the connecting rod as the research object, this paper chose the figure 4 nodes in J009 active rod, the driving rod is thrust rod of the hydraulic cylinder.

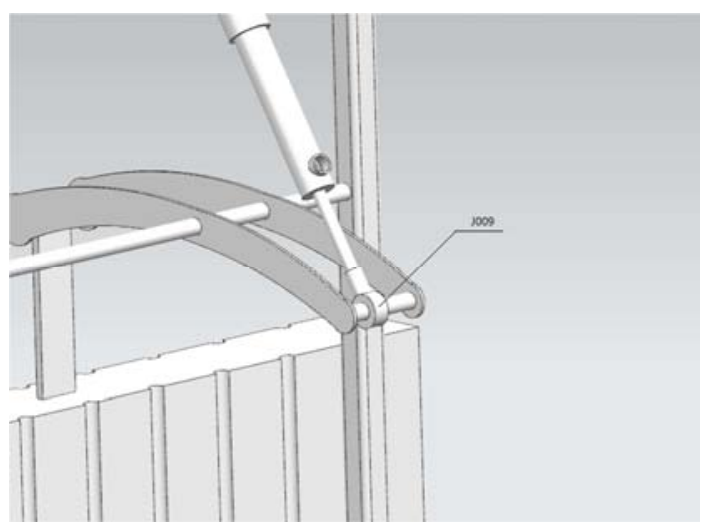

Figure 7. Node stress analysis.

Figure 8 is a node of J009 stress function, the abscissa is time Time (SEC), Force $(\mathrm{N})$ as the ordinate. On the abscissa is the time node from 0 seconds to 20 seconds. 


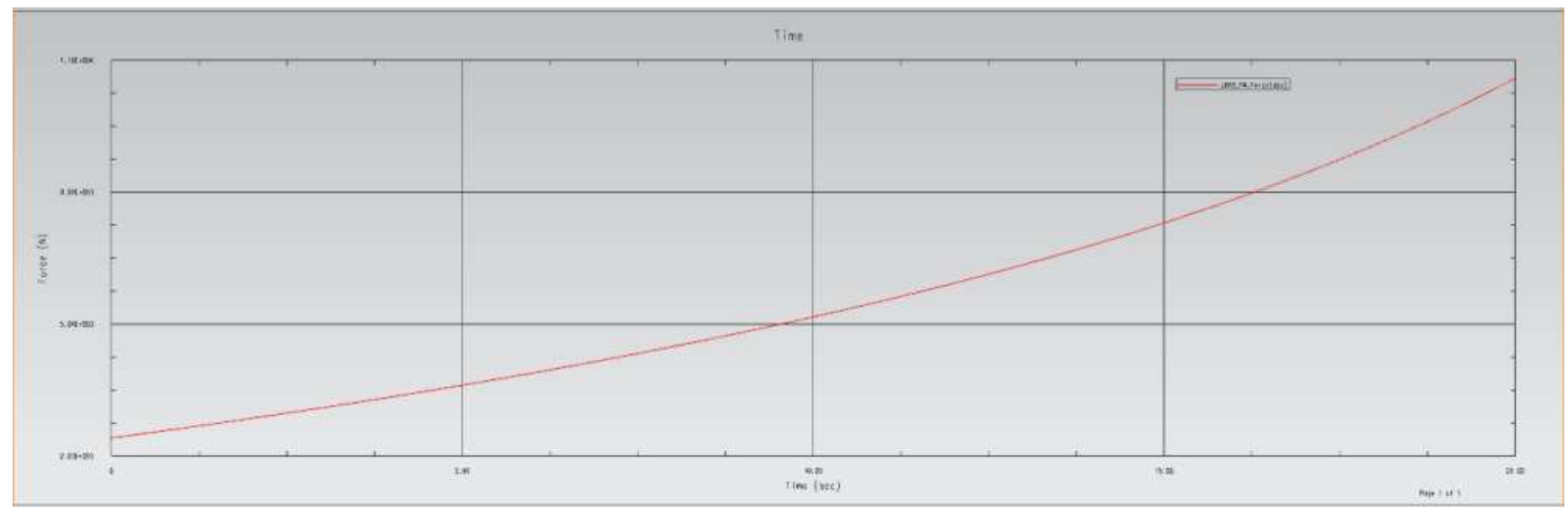

Figure 8. J009 node force curve.

As shown by the above function curve, longitudinal coordinate J009 node Force $(\mathrm{N})$ are respectively: 2.03E $+003,5.04 \mathrm{E}+003$, $8.04 \mathrm{E}+003,1.10 \mathrm{E}+004$. through the peak detection mode, as shown in figure 9 , cut the maximum force on both sides during the movement of the connecting rod is $1.064 \mathrm{E}+004 \mathrm{~N}$, namely $10640 \mathrm{~N}$.

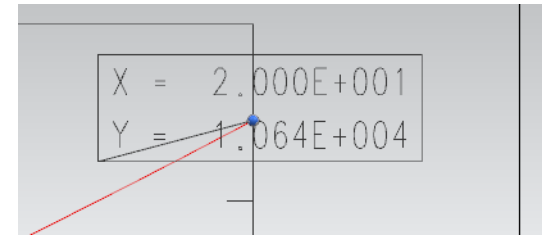

Figure 9. Node stress peak.

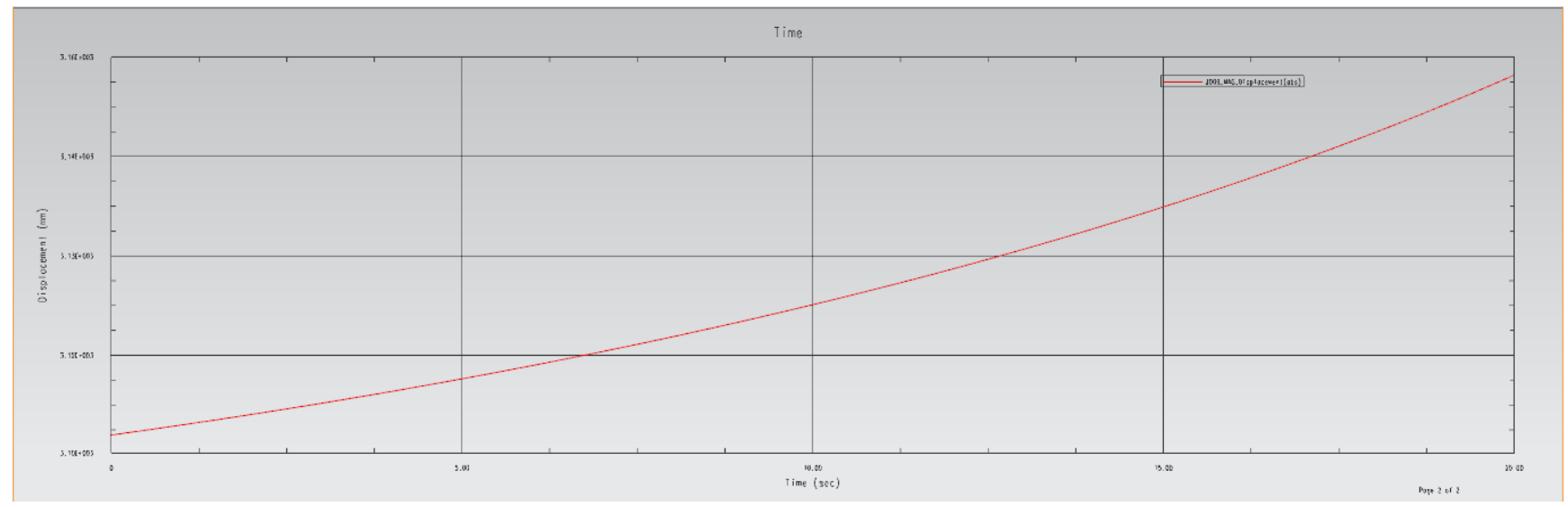

Figure 10. Gate displacement curve.

Select the displacement in the motion model of the chart component selection request, select absolute amplitude on the $\mathrm{Y}$ axis, such as generating gate displacement amplitude curves shown in figure 10, because the trajectory of the gate is the upward movement, the amplitude of displacement can be seen is gradually increased, the longitudinal numerical coordinates

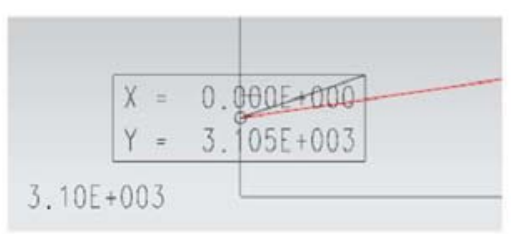

(a) were: $\quad 3.1 \mathrm{E}+003, \quad 3.12 \mathrm{E}+003, \quad 3.13 \mathrm{E}+003, \quad 3.14 \mathrm{E}+003$ $3.16 \mathrm{E}+003$, and through the peak detection mode, cut the maximum figure 11 (b): $3.155 \mathrm{E}+003$, the minimum figure (a) $3.105 \mathrm{E}+00$ By 3 we can get the maximum and minimum amplitude, the gate displacement in the movement process of the total: $31553105=50 \mathrm{~mm}$

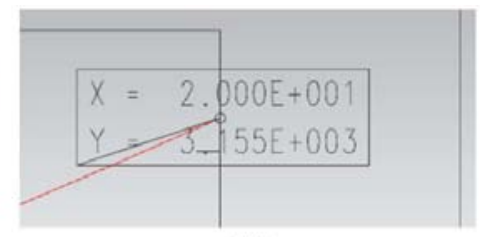

(b)

Figure 11. The displacement amplitude of the extremum. 


\section{Conclusion}

The paper designs a new type of hydraulic gate lifting device innovation, hand-operated door lifting device by hydraulic device to replace now in common use, movement of the lifting device is simulated by simulation calculation, the connecting rod by the force and displacement, improved reliability experiment through the gate of hydraulic lifting device, not only improves the safety during drying the efficiency and the drying room door landing, convenient for the operation of workers, improve the operation environment. At the same time, save energy, improve the drying room drying operation flexibility [10].

\section{References}

[1] Du Guoxing, Li Dagang wood drying technology [M] Beijing: China Forestry Press, 2005:1-2,38-40.

[2] Zhang Biguang, Xie Yongqun. Wood drying the present situation and developing trend of [J]. drying technology and equipment, 2006,4 (1): 7-14.

[3] Jianmin. Wood drying science [M] Beijing: Science Press, 2008:78-83.

[4] Lin Weekee, Li Jie, van Shaozhan. Two kinds of wood drying pit type structure analysis of [J]. wood industry, 1993,7 (3): $18-22$.

[5] Zhuang Shouzeng. Of China's wood drying technology innovation and development on [J]. forestry industry, 2008,35 (2): 6-9.

[6] Gu Lian Zhuang 100, Shouzeng. Present situation of wood industry and technology requirement of [J]. of China's wood industry, 2009,23 (3): 24-27.

[7] Zhang Liping. Design and application of hydraulic transmission system [M]. Beijing: Chemical Industry Press, 2010:3-13.

[8] Tang Jinsong. Mechanical design [M]. Shanghai: Shanghai Science Press, 1994.
[9] Chen Bingbing, Susong Joe. The hydraulic cylinder buffer structure analysis and calculation of $[\mathrm{J}]$. mechanical and electrical engineering, 1999, (5): 239-241.

[10] Zhang Jianmin. Iran. Biguang. [C]-2009 in pine Hu Chuankun wood of China's energy-saving drying technology.

[11] Yan Ping, Cao Wei-wu, Shi Sheng-hui. The application research of the new airflow guide system of wood drying chamber [J].Journal of Shanghai University of Science and Technology. 2012 34(5):487-490.

[12] Bogdan Bedelean. Comparative analysis between a drying kiln designed with and without top baffles[J]. Indian Acad Wood, 2015, 11(1):39-44.

[13] S. Sandoval-Torres et al. Numerical Simulation of Warm-air Drying of Mexican Softwood [J]. An Empirical and Mechanistic Approach, 2015, 28(1):125-133.

\section{Biography}

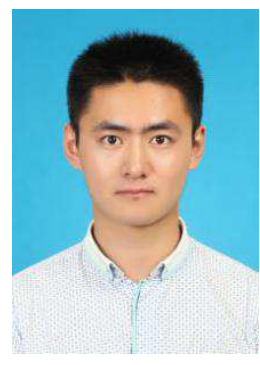

GuangFei Wang was born in August 1993 in Jiangsu Province. 2015 master's degree in mechanical manufacturing and automation, Jiangsu University of Science and now he is a postgraduate in Shanghai University of Engineering Science.

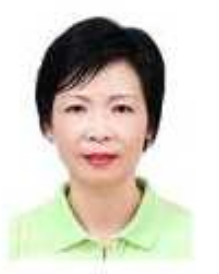

Ping Yan graduated from Tongji University and is a senior engineer of Shanghai University of Engineering Science. She has long been engaged in the design and manufacture of power machinery, the design and automatic control of energy-saving devices. 